\title{
On the Oscillations of Nonlinear Third Order Neutral Differential Equations
}

El-Sheikh MMA*, Sallam RA and Salem S

Department of Mathematics, Faculity of Science, Menofia University, Shebin El-Koom, Egypt

\begin{abstract}
This paper is concerned with the oscillation of solutions of a class of third order nonlinear neutral differential equations. New sufficient conditions guarantee that every solution is either oscillatory or tends to zero are given. The obtained results improve some recent published results in the literature. Some illustrative examples are given.
\end{abstract}

Keywords: Oscillations; Nonlinear; Integers

\section{Introduction}

In this paper, we are concerned with the oscillatory behavior of solutions of third order differential equations of the type

$$
\left(a(t)\left[z^{\prime \prime}(t)\right]^{\gamma}\right)^{\prime}+_{i=1}^{m} f_{i}\left(t, x\left(\sigma_{i}(t)\right)\right)=0, t \geq t_{0},
$$

where $z(t)=x(t)+_{j=1}^{n} p_{j}(t) x\left(\tau_{j}(t)\right), m, n$ are positive integers, $t_{0}>0$ and $a(t), p_{j}(t), \tau_{j}(t), \sigma_{i}(t) \in C\left(\left[t_{0}, \infty\right)\right), i=1,2, \ldots, m, j=1,2, \ldots, n$.

In the sequel, we assume the following conditions:

1. $a(t), p_{j}(t), \tau_{j}(t), \sigma_{i}(t)$ are positive functions, $\gamma \geq 1$ is a quotient of odd positive integers;

2. $\tau_{j}(t) \leq t, \quad \lim _{t \rightarrow \infty} \tau_{j}(t)=\infty, \quad \lim _{t \rightarrow \infty} \sigma_{i}(t)=\infty, \quad 0 \leq p_{j}(t) \leq p_{0 j} \quad$ and ${ }_{j=1}^{n} p_{0 j}<1, j=1,2, . ., n, j=1,2, . ., n$;

3. $f_{i}(t, u) \in C\left(\left[t_{0}, \infty\right) \times \mathbb{R}, \mathbb{R}\right)$ satisfies $u f_{i}(t, u)>0$ for all $u \neq 0$ and there exist positive continuous functions $q_{i}(t)$ defined on $\left[t_{0}, \infty\right)$ such that $\left|f_{i}(t, u)\right| \geq q_{i}(t)|u|^{\gamma}, i=1,2, \ldots, m$.

The study of the oscillatory behavior of solutions of third-order differential equations has recieved great interest in the last few decades. One of the reasons for that is because in the real life, during the study of some physical phenomena, the qualitative behavior of solutions of third-order differential equations can be succesfully used to predict dynamic behavior of solutions of third-order partial differential equations.

Following this trend we are concerned in this paper with the oscillatory behavior of the third-order neutral differential equation (E).

By a solution of (E), we mean a function $x \in C\left(\left[T_{x}, \infty\right), \mathbb{R}\right), T_{x} \geq t_{0}$, which has the properities $z \in C^{2}\left(\left[T_{x}, \infty\right), R\right), a(t)\left[z^{\prime \prime}(t)\right]^{\gamma} \in C^{1}\left(\left[T_{x}, \infty\right), R\right)$ and satisfies (E) on $\left[T_{x}, \infty\right)$. In this paper, we consider only those solutions $x$ of (E) which satisfy $\sup \{|x(t)|: t \geq T\}>0$ for all $T \geq T_{x}$. A solution of (E) is called oscillatory if it has arbitrarily large zeros on $\left[T_{x}, \infty\right)$; otherwise it is called nonoscillatory.

Recently, increasing attention has been devoted to the oscillation of differential equations of the form (E) and some of its exceptions; have been the subject of intensive researchs see for example the papers [1-13] and references cited in. In particular, we mention here the paper of Grace et al. [5] which studied the oscillation of the third order delay differential equation.

$$
\left(a(t)\left[x^{\prime \prime}(t)\right]^{\gamma}\right)^{\prime}+q(t) f(x(\tau(t)))=0, t \geq t_{0},
$$

By comparing with the first order delay equation, where in their comparison principle it is always required that $\tau(t)<t$. More recently, Baculková and Džurina [3] improved their results for the case when

$$
{ }_{t_{0}}^{\infty} a^{\frac{-1}{\gamma}}(t) d t=\infty .
$$

While the same authors, Baculková and Džurina [2] discussed the oscillation behavior of eqn. (1.1) in the case when

$$
{ }_{t_{0}}^{\infty} a^{\frac{-1}{\gamma}}(t) d t<\infty .
$$

Zhong et al. [13] adapted Grace et al.'s method and extended some of their results to the neutral differential equation

$$
\left(a(t)\left[(x(t)+p(t) x(\sigma(t)))^{\prime \prime}\right]^{\gamma}\right)^{\prime}+q(t) f(x(\tau(t)))=0 .
$$

However, the results [13] cannot be applied when

$$
{ }_{t_{0}}^{\infty} a^{\frac{-1}{\gamma}}(t) d t<\infty \text { and } \tau(t) \geq t \text {. }
$$

In this paper are cocerned with this gap for the more general equation (E) by applying a technique similar to that given by those of refs. [8] and [10].

\section{Preliminaries}

\section{Lemma 1}

Let $x(t)$ be a positive solution of eqns. (E) and (1.2) holds. Then there are only one of the following two cases:

$$
\begin{aligned}
& \text { (I) } z(t)>0, z^{\prime}(t)>0, z^{\prime \prime}(t)>0 \text { and }\left(a(t)\left[z^{\prime \prime}(t)\right]^{\gamma}\right)^{\prime}<0 ; \\
& \text { (II) } z(t)>0, z^{\prime}(t)<0, z^{\prime \prime}(t)>0 \text { and }\left(a(t)\left[z^{\prime \prime}(t)\right]^{\gamma}\right)^{\prime}<0,
\end{aligned}
$$

for $t \geq t_{1}$, where $t_{1} \geq t_{0}$ is sufficiently large.

Proof: The proof is similar to the proof of Lemma 1 [1] and so it is omitted.

*Corresponding author: El-sheikh MMA, Professor, Department of Mathematics Faculity of Science, Shebeen El-Kom, Egypt, Tel: +20 48 2222170; E-mail: msheikh_1999@yahoo.com

Received May 12, 2017; Accepted September 20, 2017; Published September 27, 2017

Citation: El-Sheikh MMA, Sallam RA, Salem S (2017) On the Oscillations of Nonlinear Third Order Neutral Differential Equations. J Appl Computat Math 6: 361. doi: 10.4172/2168-9679.1000361

Copyright: @ 2017 El-Sheikh MMA, et al. This is an open-access article distributed under the terms of the Creative Commons Attribution License, which permits unrestricted use, distribution, and reproduction in any medium, provided the original author and source are credited. 


\section{Lemma 2}

Let $x(t)$ be a positive solution of (E). Suppose further that (1.2) holds and the corresponding $z(t)$ satisfies case (II) in Lemma 1. If

$$
\underset{t_{0} v}{\infty}\left[\frac{1}{a(u)} \sum_{i=1}^{m} q_{i}(s) d s\right]^{\gamma} d u d v=\infty
$$

then $\lim _{t \rightarrow \infty} x(t)=0$.

Proof: Assume that $x(t)$ is a positive solution of $(\mathrm{E})$. It is clear that there exists a finite limit, say $\lim _{t \rightarrow \infty} z(t)=l \geq 0$. We claim that $l=0$. If not, then for any $\varepsilon>0$, there exists a $t_{1} \geq t_{0}$ such that $l<z(t)<l+\varepsilon$. By choosing $0<\varepsilon<l\left(1-\sum_{j=1}^{n} p_{0 j}\right) / \sum_{j=1}^{n} p_{0 j}$, we get

$$
\begin{aligned}
& x(t)=z(t)-\sum_{j=1}^{n} p_{j}(t) x\left(\tau_{j}(t)\right) \\
& >l-\sum_{j=1}^{n} p_{j}(t) z\left(\tau_{j}(t)\right) \\
& >l-\sum_{j=1}^{n} p_{0 j}(l+\varepsilon)=N(l+\varepsilon)>N z(t)
\end{aligned}
$$

where $N=\left(l-\sum_{j=1}^{n} p_{0 j}(l+\varepsilon)\right) /(l+\varepsilon)$. This with (E) in view of $\left(\mathrm{A}_{3}\right)$ leads to

$$
\begin{aligned}
& 0=\left(a(t)\left[z^{\prime \prime}(t)\right]^{\gamma}\right)^{\prime}++_{i=1}^{m} f_{i}\left(t, x\left(\sigma_{i}(t)\right)\right) \\
& \geq\left(a(t)\left[z^{\prime \prime}(t)\right]^{\gamma}\right)^{\prime}+N_{i=1}^{m} q_{i}(t) z^{\gamma}\left(\sigma_{i}(t)\right)
\end{aligned}
$$

Integrating from $t$ to $\infty$ and using the fact that $z(t)>l$, we obtain

$$
0 \geq-a(t)\left[z^{\prime \prime}(t)\right]^{\gamma}+(N l)^{\gamma} \sum_{i=1}^{m} \int_{t}^{\infty} q_{i}(s) d s \text {. }
$$

i.e.,

$$
z^{\prime \prime}(t) \geq(N l)\left[\frac{1}{a(t)} \sum_{i=1}^{m} \int_{t}^{\infty} q_{i}(s) d s\right]^{\frac{1}{\gamma}} .
$$

Again by integrating eqn. (2.4) from $t$ to $\infty$, we get

$$
-z^{\prime}(t) \geq N l \int_{t}^{\infty}\left[\frac{1}{a(u)} \sum_{i=1}^{m} \int_{u}^{\infty} q_{i}(s) d s\right]^{\frac{1}{\gamma}} d u \text {. }
$$

Integrating eqn. (2.5) from $t_{1}\left(t_{1} \geq t_{0}\right)$ to $\infty$, it follows that

$$
\frac{z\left(t_{1}\right)}{N l} \geq \int_{t_{1}}^{\infty} \int_{v}^{\infty}\left[\frac{1}{a(u)} \sum_{i=1}^{m} \int_{u}^{\infty} q_{i}(s) d s\right]^{\frac{1}{\gamma}} d u d v .
$$

This contradicts eqn. (2.1). Hence $l=0$. But since $0 \leq x(t) \leq z(t)$, then $\lim _{t \rightarrow \infty} z(t)=\lim _{t \rightarrow \infty} x(t)=0$. This completes the proof.

Now we outline the following two lemmas [1].

\section{Lemma 3}

Assume that $u(t)>0, u^{\prime}(t)>0$ and $u^{\prime \prime}(t) \leq 0$, for $t \geq t_{0}$. If $\sigma \in C\left(\left[t_{0}, \infty\right),(0, \infty)\right), \sigma(t) \leq t$ and $\lim _{t \rightarrow \infty} \sigma(t)=\infty$,

Proof: Then for every, there exists a $T_{\alpha} \geq t_{0}$ such that $u(\sigma(t)) / \sigma(t) \geq \alpha u(t) / t$ for $t \geq T_{\alpha}$.

\section{Lemma 4}

Assume that $u(t)>0, u^{\prime}(t)>0$, and $u^{\prime \prime \prime}(t) \leq 0$, for $t \geq t_{0}$. Then for each $\beta \in(0,1)$, there exists a $T_{\beta} \geq t_{0}$ such that $u(t) \geq \beta t u^{\prime}(t) / 2$ for $t \geq T_{\beta}$.

Further, we give the following auxiliary result which is extracted from those [6] and [7].

\section{Lemma 5}

Let $\gamma \geq 1$ be a ratio of two odd positive numbers. Then,

$$
A^{1+\frac{1}{\gamma}}-(A-B)^{1+\frac{1}{\gamma}} \leq \frac{B^{\frac{1}{\gamma}}}{\gamma}[(\gamma+1) A-B], \text { forall } A B \geq 0
$$

and

$$
C^{1+\frac{1}{\gamma}}-\frac{\gamma+1}{\gamma} C D^{\frac{1}{\gamma}} \geq \frac{-1}{\gamma} D^{1+\frac{1}{\gamma}}, \text { forany } C, D \geq 0 .
$$

\section{Main Results}

In this section, we establish new oscillation criteria for eqn. (E) by using a generalized Riccati transformation and integral averaging technique of Philos-type [12]. Let

$$
D=\left\{(t, s): t \geq s \geq t_{0}\right\} \text { and } D_{0}=\left\{(t, s): t>s \geq t_{0}\right\} .
$$

A function $H \in C^{1}(D, \mathbb{R})$ is said to belong to the class $X_{\gamma}$ if

1. $H(t, t)=0$ and $H(t, s)>0$ for all $(t, s) \in D_{0}$;

2. $H$ has a nonpositive continuous partial derivative $\partial H / \partial s$ on $D_{0}$ with respect to the second variable and there exist functions $\rho \in C^{1}\left(\left[t_{0}, \infty\right),(0, \infty)\right), \varphi \in C^{1}\left(\left[t_{0}, \infty\right),(0, \infty)\right)$ and $h \in C\left(D_{0}, \mathbb{R}\right)$ such that

$$
\frac{\partial H(t, s)}{\partial s}+\left[\frac{\rho^{\prime}(s)}{\rho(s)}+(\gamma+1) \varphi^{\frac{1}{\gamma}}(s)\right] H(t, s)=-h(t, s)(H(t, s))^{\frac{\gamma}{\gamma+1}} .
$$

Note that for $\gamma=1 . X_{\gamma}$ reduces to the class of functions $X$ used [8]. For $\rho=1$ and $\varphi=0, X_{\gamma}$ reduces to the class of functions $W_{\gamma}$ used [9].

\section{Theorem 6}

Suppose that the conditions $\left(\mathrm{A}_{1}\right)-\left(\mathrm{A}_{3}\right)$ hold, $\sigma_{i}(t) \leq t$, for $i=1,2, \ldots, m$, eqn. (1.2) and (2.1) be satisfied. Assume further that

$$
\lim _{t \rightarrow \infty} \sup \frac{1}{H\left(t, t_{0}\right)} \int_{t_{0}}^{t}\left[H(t, s) G(s)-\frac{\left(h_{-}(t, s)\right)^{\gamma+1} a(s) \rho(s)}{(\gamma+1)^{\gamma+1}}\right] d s=\infty
$$

holds for some $c \in(0,1)$ and for some $H \in X_{\gamma}$, where

$$
\begin{aligned}
& G(t)=\rho(t)\left[\left(\frac{1}{2} c\left(1-{ }_{j=1}^{n} p_{0 j}\right)\right)_{i=1}^{\gamma m} q_{i}(t)\left(\frac{\left(\sigma_{i}(t)\right)^{2}}{t}\right)^{\gamma}+a(t) \varphi^{\frac{\gamma+1}{\gamma}}(t)-(a(t) \varphi(t))^{\prime}\right] \\
& \text { and } h_{-}(t, s)=\max \{0,-h(t, s)\} .
\end{aligned}
$$

Then every solution $x$ of (E) is either oscillatory or satisfies $\lim _{t \rightarrow \infty} x(t)=0$.

Proof: Assume that $x(t)$ is a non-oscillatory solution of eqn. (E). Without loss of generality, we may assume that $x(t)$ is eventually positive. Then, there exists a $t_{1} \geq t_{0}$ such that $x(t)>0, x\left(\tau_{j}(t)\right)>0$ and $x\left(\sigma_{i}(t)\right)>0$ for $t \geq t_{1}$ and $i=1,2, . ., m, j=1,2, . ., n$. By Lemma 1 , it follows that $z$ satisfies either (I) or (II) for $t \geq t_{2}$, where $t_{2} \geq t_{1}$ is large enough. We consider each of the two cases separately. Assume first that case (I) holds. Hence since $z^{\prime}(t)>0$, we have 
$x(t)=z(t)-{ }_{j=1}^{n} p_{j}(t) x\left(\tau_{j}(t)\right)$

$\geq z(t)-_{j=1}^{n} p_{0 j} z(t)=z(t)\left(1--_{j=1}^{n} p_{0 j}\right)$

In view of $(\mathrm{E})$, we have

$$
\begin{aligned}
& \left(a(t)\left[z^{\prime \prime}(t)\right]^{\gamma}\right)^{\prime}=--_{i=1}^{m} f_{i}\left(t, x\left(\sigma_{i}(t)\right)\right) \leq-{ }_{i=1}^{m} q_{i}(t) x^{\gamma}\left(\sigma_{i}(t)\right) \\
& \leq-\left(1-{ }_{j=1}^{n} p_{0 j}\right)_{i=1}^{\gamma m} q_{i}(t) z^{\gamma}\left(\sigma_{i}(t)\right)
\end{aligned}
$$

Now, consider a generalized Riccati substitution of the form

$\omega(t)=\rho(t) a(t)\left[\left(\frac{z^{\prime \prime}(t)}{z^{\prime}(t)}\right)^{\gamma}+\varphi(t)\right], t \geq t_{2}$.

Then by eqn. (3.6), we get

$$
\begin{aligned}
& \omega^{\prime}(t) \leq \frac{\rho^{\prime}(t)}{\rho(t)} \omega(t)+\rho(t)(a(t) \varphi(t))^{\prime}-\left(1-{ }_{j=1}^{n} p_{0 j}\right)^{\gamma} \rho(t)_{i=1}^{m} \frac{q_{i}(t) z^{\gamma}\left(\sigma_{i}(t)\right)}{z^{\prime \gamma}(t)} \\
& -\gamma \rho(t) a(t)\left[\frac{\omega(t)}{a(t) \rho(t)}-\varphi(t)\right]^{\frac{\gamma+1}{\gamma}}
\end{aligned}
$$

Therefore from Lemma 4 and Lemma 5, it follows that, for any $\alpha \in(0,1)$ and $\beta \in(0,1)$, we have

$$
\frac{z\left(\sigma_{i}(t)\right)}{z^{\prime}(t)}=\frac{z\left(\sigma_{i}(t)\right)}{z^{\prime}\left(\sigma_{i}(t)\right)} \frac{z^{\prime}\left(\sigma_{i}(t)\right)}{z^{\prime}(t)} \geq \frac{\alpha \beta}{2} \frac{\left(\sigma_{i}(t)\right)^{2}}{t}, i=1,2, . ., m .
$$

Thus

$$
\left(\frac{z\left(\sigma_{i}(t)\right)}{z^{\prime}(t)}\right)^{\gamma} \geq\left[\frac{\alpha \beta}{2} \frac{\left(\sigma_{i}(t)\right)^{2}}{t}\right]^{\gamma}, i=1,2, . ., m .
$$

Combining eqns. (3.8) and (3.9), we get

$$
\begin{aligned}
& \omega^{\prime}(t) \leq \frac{\rho^{\prime}(t)}{\rho(t)} \omega(t)-\left[\frac{1}{2} c\left(1-{ }_{j=1}^{n} p_{0 j}\right)\right]^{\gamma} \rho(t)_{i=1}^{m} q_{i}(t)\left(\frac{\left(\sigma_{i}(t)\right)^{2}}{t}\right)^{\gamma} \\
& +\rho(t)(a(t) \varphi(t))^{\prime}-\gamma \rho(t) a(t)\left[\frac{\omega(t)}{a(t) \rho(t)}-\varphi(t)\right]^{\frac{\gamma+1}{\gamma}},
\end{aligned}
$$

where $c=\alpha \beta$. Applying the inequality eqn. (2.6) of Lemma 3 with $A=\frac{\omega(t)}{a(t) \rho(t)}$ and $B=\varphi(t)$, we get

$$
\left.\frac{\omega(t)}{\imath(t) \rho(t)}-\varphi(t)\right]^{\frac{\gamma+1}{\gamma}} \geq\left[\frac{\omega(t)}{a(t) \rho(t)}\right]^{\frac{\gamma+1}{\gamma}}-\frac{\varphi^{\frac{1}{\gamma}}(t)}{\gamma}\left[\frac{(\gamma+1) \omega(t)}{a(t) \rho(t)}-\varphi(t)\right]
$$

This with eqn. (3.3) yields

$$
\begin{aligned}
& \omega^{\prime}(t) \leq \frac{\rho^{\prime}(t)}{\rho(t)} \omega(t)-\left[\frac{1}{2} c\left(1-{ }_{j=1}^{n} p_{0 j}\right)\right]^{\gamma} \rho(t)_{i=1}^{m} q_{i}(t)\left(\frac{\left(\sigma_{i}(t)\right)^{2}}{t}\right)^{\gamma} \\
& +\rho(t)(a(t) \varphi(t))^{\prime}-\frac{\gamma \omega^{\frac{\gamma+1}{\gamma}}(t)}{(a(t) \rho(t))^{\frac{1}{\gamma}}}+(\gamma+1) \varphi^{\frac{1}{\gamma}}(t) \omega(t) \\
& -a(t) \rho(t) \varphi^{\frac{\gamma+1}{\gamma}}(t) \\
& =-\rho(t)\left[\left(\frac{1}{2} c\left(1-{ }_{j=1}^{n} p_{0 j}\right)\right]_{i=1}^{\gamma m} q_{i}(t)\left(\frac{\left(\sigma_{i}(t)\right)^{2}}{t}\right)^{\gamma}+a(t) \varphi^{\frac{\gamma+1}{\gamma}}(t)-(a(t) \varphi(t))^{\prime}\right] \\
& +\left[\frac{\rho^{\prime}(t)}{\rho(t)}+(\gamma+1) \varphi^{\frac{1}{\gamma}}(t)\right] \omega(t)-\frac{\gamma \omega^{\frac{\gamma+1}{\gamma}}(t)}{(a(t) \rho(t))^{\frac{1}{\gamma}}} .
\end{aligned}
$$

i.e.

$$
\omega^{\prime}(t) \leq-G(t)+\psi(t) \omega(t)-\frac{\gamma \omega^{\frac{\gamma+1}{\gamma}}(t)}{(a(t) \rho(t))^{\frac{1}{\gamma}}},
$$

where $\psi(t)=\left[\frac{\rho^{\prime}(t)}{\rho(t)}+(\gamma+1) \varphi^{\frac{1}{\gamma}}(t)\right]$. Replacing $t$ in the place of $s$ eqn. (3.12), multiplying both sides by $H(t, s)$ and integrating with respect to $s$ using eqns. (3.1) and (3.4), we get

$$
\begin{aligned}
& { }_{T}^{t} H(t, s) G(s) d s \leq H(t, T) \omega(T)+{ }_{T}^{t} \frac{\partial H(t, s)}{\partial s} \omega(s) d s+_{T}^{t} H(t, s) \psi(s) \omega(s) d s \\
& --_{T}^{t} \frac{\gamma H(t, s)}{(a(s) \rho(s))^{\frac{1}{\gamma}}} \omega^{\frac{\gamma+1}{\gamma}}(s) d s \\
& =H(t, T) \omega(T)+_{T}^{t}\left[-h(t, s)(H(t, s))^{\frac{\gamma}{\gamma+1}} \omega(s)-\frac{\gamma H(t, s)}{(a(s) \rho(s))^{\frac{1}{\gamma}}} \omega^{\frac{\gamma+1}{\gamma}}(s)\right] d s \\
& \leq H(t, T) \omega(T)+_{T}^{t}\left[h_{-}(t, s)(H(t, s))^{\frac{\gamma}{\gamma+1}} \omega(s)-\frac{\gamma H(t, s)}{(a(s) \rho(s))^{\frac{1}{\gamma}}} \omega^{\frac{\gamma+1}{\gamma}}(s)\right] d s .
\end{aligned}
$$

Now define

$$
C=\left(\frac{\gamma H(t, s) \omega^{\frac{\gamma+1}{\gamma}}(s)}{(a(s) \rho(s))^{\frac{1}{\gamma}}}\right)^{\frac{\gamma}{\gamma+1}} \text { and } D=\left(\frac{h_{-}(t, s)(\gamma a(s) \rho(s))^{\frac{1}{\gamma+1}}}{(\gamma+1)}\right)^{\gamma} \text {. }
$$

Applying the inequality eqn. (2.7) of Lemma 3, we get

$$
h_{-}(t, s)(H(t, s))^{\frac{\gamma}{\gamma+1}} \omega(s)-\frac{\gamma H(t, s) \omega^{\frac{\gamma+1}{\gamma}}(s)}{(a(s) \rho(s))^{\frac{1}{\gamma}}} \leq \frac{\left(h_{-}(t, s)\right)^{\gamma+1} a(s) \rho(s)}{(\gamma+1)^{\gamma+1}} .
$$

Thus by eqns. (3.13) and (3.14), we have

$$
\frac{1}{H(t, T)_{T}}{ }_{T}^{t}\left[H(t, s) G(s)-\frac{\left(h_{-}(t, s)\right)^{\gamma+1} a(s) \rho(s)}{(\gamma+1)^{\gamma+1}}\right] d s \leq \omega(T) .
$$

This contradicts eqn. (3.2). Now consider the case (II) in Lemma 1. Then by Lemma 2 , we have $\lim _{t \rightarrow \infty} x(t)=0$. This completes the proof.

In the following result, we consider the case when (1.3) holds, where in this case, there is one more possibility other than (I) and (II) mentioned in Lemma 1.

\section{Theorem 7}

Let all the assumptions of Theorem 6 be satisfied. Suppose further that the condition (1.2) is replaced by (1.3) for some $c \in(0,1)$ and for some $H \in X_{\gamma},(3.2)$ holds. If for $\rho(t)=1, \varphi(t)=0$, there exists a function $H_{1}(t, s) \in X_{\gamma}$ such that,

$$
\limsup _{t \rightarrow \infty} \frac{1}{H_{1}\left(t, t_{2}\right)_{t_{2}}}\left[\left(1-{ }_{j=1}^{n} p_{0 j}\right)^{\gamma} H_{1}(t, s)_{i=1}^{m} q_{i}(s)\left(\sigma_{i}(s)-t_{1}\right)^{\gamma}-\frac{\left(h_{1}(t, s)\right)^{\gamma+1} a(s)}{(\gamma+1)^{\gamma+1}}\right] d s>0
$$

for all sufficiently large $t_{1} \geq t_{0}$ and $t_{2} \geq t_{1} \geq t_{0}$, then every solution $x$ of (E) is either oscillatory or satisfies $\lim _{t \rightarrow \infty} x(t)=0$.

Proof: Assume that $x(t)$ is a positive solution of $(\mathrm{E})$. Based on the condition eqn. (1.3), there exist three possible cases (I), (II) (as those of Lemma 1), and the following third possibility

(III) $z(t)>0, z^{\prime}(t)>0, z^{\prime \prime}(t)<0,\left(a(t)\left[z^{\prime \prime}(t)\right]^{\gamma}\right)^{\prime}<0$, for $t \geq t_{1}, t_{1}$ is large enough.

Firstly if case (I) and case (II) hold, respectively, we can obtain the conclusion of Theorem 7 by applying the proof of theorem 6 . 
Now assume the case (III) holds. Then, $z^{\prime \prime}(t)<0$ for all $t \geq t_{1}$. Define the function

$$
\phi(t)=\frac{a(t)\left(z^{\prime \prime}(t)\right)^{\gamma}}{z^{\prime \prime}(t)}, t \geq t_{1} .
$$

It is clear that $\phi(t)<0$ for $t \geq t_{1}$, and

$$
\phi^{\prime}(t)=\frac{\left(a(t)\left(z^{\prime \prime}(t)\right)^{\gamma}\right)^{\prime}}{z^{\prime \prime}(t)}-\frac{\gamma a(t)\left(z^{\prime \prime}(t)\right)^{\gamma+1}}{z^{\prime \gamma+1}(t)} \text {. }
$$

Since $z^{\prime}(t)>0$, then by eqns. (3.5) and (3.17) in view of (E), we get

$$
'(t) \leq-\left(1-{ }_{j=1}^{n} p_{0 j}\right){ }_{i=1}^{m} q_{i}(t)\left(\frac{z(\sigma(t))}{z(t)}\right)-\frac{\gamma \phi \overline{(t)}}{a^{-}(t)} .
$$

In view of (III), we have

$z(t) \geq\left(t-t_{1}\right) z^{\prime}(t)$.

Hence,

$\left(\frac{z(t)}{t-t_{1}}\right)^{\prime} \leq 0$.

This implies that

$$
\frac{z\left(\sigma_{i}(t)\right)}{z(t)} \geq \frac{\sigma_{i}(t)-t_{1}}{t-t_{1}}, i=1,2, . ., m \text {. }
$$

This with eqn. (3.19), leads to

$$
\frac{z\left(\sigma_{i}(t)\right)}{z^{\prime}(t)} \geq\left(\sigma_{i}(t)-t_{1}\right) \text {. }
$$

Substituting in eqn. (3.18), we get

$$
\phi^{\prime}(t) \leq-\left(1-{ }_{j=1}^{n} p_{0 j}\right)_{i=1}^{\gamma m} q_{i}(t)\left(\sigma_{i}(t)-t_{1}\right)^{\gamma}-\frac{\gamma \phi^{\frac{\gamma+1}{\gamma}}(t)}{a^{\frac{1}{\gamma}}(t)} \text {. }
$$

Interchanging $t$ with $s$ in eqn. (3.22), multiplying both sides by $H_{1}(t, s)$ and integrating with respect to $s$ from $t_{2}$ to $t\left(t_{2} \geq t_{1}\right)$. Then in view of the properties of $H(t, s)$ with $\rho(t)=1, \varphi(t)=0$ it follows that,

$$
\begin{aligned}
& { }_{t_{2}}^{t} H_{1}(t, s)\left(1-{ }_{j=1}^{n} p_{0 j}\right)_{i=1}^{\gamma m} q_{i}(s)\left(\sigma_{i}(s)-t_{1}\right)^{\gamma} \leq H_{1}\left(t, t_{2}\right) \phi\left(t_{2}\right)+{ }_{t_{2}}^{t} \frac{\partial H_{1}(t, s)}{\partial s} \phi(s) d s \\
& -{ }_{t_{2}}^{t} \frac{\gamma \phi^{\frac{\gamma+1}{\gamma}}(s) H_{1}(t, s)}{a^{\frac{1}{\gamma}}(s)} d s \\
& \leq H_{1}\left(t, t_{2}\right) \phi\left(t_{2}\right)+_{t_{2}}^{t}-h_{1}(t, s)\left(H_{1}(t, s)\right)^{\frac{\gamma}{\gamma+1}} \phi(s) d s \\
& +_{t_{2}}^{t}\left[-\frac{\gamma H_{1}(t, s)}{a^{\frac{1}{\gamma}}(s)}(-\phi(s))^{\frac{\gamma+1}{\gamma}}\right] d s
\end{aligned}
$$

Now define

$$
C_{1}^{\frac{\gamma+1}{\gamma}}=\frac{\gamma H_{1}(t, s)(-\phi(s))^{\frac{\gamma+1}{\gamma}}}{a^{\frac{1}{\gamma}}(s)} \text { and } D_{1}^{\frac{1}{\gamma}}=\frac{h_{1}(t, s)(\gamma a(s))^{\frac{1}{\gamma+1}}}{(\gamma+1)} \text {. }
$$

Applying the inequality eqn. (2.7), it follows that

$$
-h_{1}(t, s)\left(H_{1}(t, s)\right)^{\frac{\gamma}{\gamma+1}} \phi(s)-\frac{\gamma H_{1}(t, s)}{a^{\frac{1}{\gamma}}(s)}(-\phi(s))^{\frac{\gamma+1}{\gamma}} \leq \frac{\left(h_{1}(t, s)\right)^{\gamma+1} a(s)}{(\gamma+1)^{\gamma+1}} \text {. (3.24) }
$$

This with eqn. (3.23) leads to

$$
\frac{1}{H_{1}\left(t, t_{2}\right)_{t_{2}}}{ }_{t_{2}}^{t}\left[\left(1-{ }_{j=1}^{n} p_{0 j}\right)^{\gamma} H_{1}(t, s)_{i=1}^{m} q_{i}(s)\left(\sigma_{i}(s)-t_{1}\right)^{\gamma}-\frac{\left(h_{1}(t, s)\right)^{\gamma+1} a(s)}{(\gamma+1)^{\gamma+1}}\right] d s \leq \phi\left(t_{2}\right),
$$

which contradicts eqn. (3.15). This completes the proof.

\section{Theorem 8}

Assume that the conditions $\left(\mathrm{A}_{1}\right)-\left(\mathrm{A}_{3}\right)$ hold. Suppose that $i=1,2, . ., m$, for $i=1,2, . ., m$, eqn. (1.2), (2.1) and (3.4) hold. If for some $\beta \in(0,1)$ and for some $H \in X_{\gamma}$,

$$
\lim _{t \rightarrow \infty} \sup \frac{1}{H\left(t, t_{0}\right)_{t_{0}}}{ }^{t}\left[H(t, s) G_{1}(s)-\frac{\left(h_{-}(t, s)\right)^{\gamma+1} a(s) \rho(s)}{(\gamma+1)^{\gamma+1}}\right] d s=\infty
$$

where

$$
G_{1}(t)=\rho(t)\left[\left(\frac{1}{2} \beta t\left(1-{ }_{j=1}^{n} p_{0 j}\right)\right)_{i=1}^{\gamma m} q_{i}(t)+a(t) \varphi^{\frac{\gamma+1}{\gamma}}(t)-(a(t) \varphi(t))^{\prime}\right],
$$

then every solution $x$ of eqn. (E) is either oscillatory or satisfies $\lim _{t \rightarrow \infty} x(t)=0$.

Proof: Assume that $x(t)$ is a non-oscillatory solution of (E). Without loss of generality, we may assume that $x(t)$ is eventually positive. Going through as in the proof of Theorem 6 , we arrive eqn. (3.8). Since $z^{\prime}(t)>0$ and $\sigma_{i}(t) \geq t$, for $i=1,2, . ., m$, we obtain

$$
\begin{aligned}
& \omega^{\prime}(t) \leq \frac{\rho^{\prime}(t)}{\rho(t)} \omega(t)+\rho(t)(a(t) \varphi(t))^{\prime}-\left(1-{ }_{j=1}^{n} p_{0 j}\right)^{\gamma} \rho(t)_{i=1}^{m} \frac{q_{i}(t) z^{\gamma}(t)}{z^{\prime \gamma}(t)} \\
& -\gamma \rho(t) a(t)\left[\frac{\omega(t)}{\rho(t) a(t)}-\varphi(t)\right]^{\frac{\gamma+1}{\gamma}}
\end{aligned}
$$

Then from Lemma 5 It follows for any $\beta \in(0,1)$ that,

$\frac{z(t)}{z^{\prime}(t)} \geq \frac{\beta t}{2}$.

i.e.,

$$
\left(\frac{z(t)}{z^{\prime}(t)}\right)^{\gamma} \geq\left(\frac{\beta t}{2}\right)^{\gamma} \text {. }
$$

Combining eqns. (3.27) and (3.28), we get

$$
\begin{aligned}
& \omega^{\prime}(t) \leq \frac{\rho^{\prime}(t)}{\rho(t)} \omega(t)+\rho(t)(a(t) \varphi(t))^{\prime}-\left(\frac{1}{2} \beta t\left(1-{ }_{j=1}^{n} p_{0 j}\right)\right)^{\gamma} \rho(t)_{i=1}^{m} q_{i}(t) \\
& -\gamma \rho(t) a(t)\left[\frac{\omega(t)}{\rho(t) a(t)}-\varphi(t)\right]^{\frac{\gamma+1}{\gamma}}
\end{aligned}
$$

Using the inequality eqns. (2.6) to (3.29), we conclude that

$$
\begin{aligned}
& \omega^{\prime}(t) \leq-\rho(t)\left[\left(\frac{1}{2} \beta t\left(1-{ }_{j=1}^{n} p_{0 j}\right)\right)_{i=1}^{\gamma m} q_{i}(t)+a(t) \varphi^{\frac{\gamma+1}{\gamma}}(t)-(a(t) \varphi(t))^{\prime}\right] \\
& +\left[\frac{\rho^{\prime}(t)}{\rho(t)}+(\gamma+1) \varphi^{\frac{1}{\gamma}}(t)\right] \omega(t)-\frac{\gamma \omega^{\frac{\gamma+1}{\gamma}}(t)}{(\rho(t) a(t))^{\frac{1}{\gamma}}} \\
& =-G_{1}(t)+\psi(t) \omega(t)-\frac{\gamma \omega^{\frac{\gamma+1}{\gamma}}(t)}{(\rho(t) a(t))^{\frac{1}{\gamma}}},
\end{aligned}
$$

where $\psi(t)=\left[\frac{\rho^{\prime}(t)}{\rho(t)}+(\gamma+1) \varphi^{\frac{1}{\gamma}}(t)\right]$. Replacing $t$ with seqn. (3.30), 
Citation: El-Sheikh MMA, Sallam RA, Salem S (2017) On the Oscillations of Nonlinear Third Order Neutral Differential Equations. J Appl Computat Math 6: 361. doi: 10.4172/2168-9679.1000361

Page 5 of 5

multiplying both sides by $H(t, s)$ and integrating with respect to $s$ from $T_{1}\left(T_{1} \geq t_{0}\right)$ to $t$. In view of the fact that $H(t, t)=0$, it follows eqns. (3.1) and (3.4) that

$$
{ }_{T_{1}}^{t} H(t, s) G_{1}(s) d s \leq H\left(t, T_{1}\right) \omega\left(T_{1}\right)+{ }_{T_{1}}^{+}\left[h_{-}(t, s)(H(t, s))^{\frac{\gamma}{\gamma+1}} \omega(s)-\frac{\gamma H(t, s)}{(a(s) \rho(s))^{\frac{1}{\gamma}}} \omega^{\frac{\gamma+1}{\gamma}}(s)\right] d s .
$$

Again following the proof of Theorem 6, we get a contradiction eqn. (3.25). Assume that case (II) holds. By virtue of Lemma 2, $\lim x(t)=0$, and thus the proof is completed.

\section{Theorem 9}

Let all the assumptions of Theorem 8 be satisfied. Suppose that the condition eqn. (1.2) is replaced by eqn. (1.3) and for some $\beta \in(0,1)$ and some $H \in X_{y}$, eqn. (3.25) holds. Suppose further that for $\rho(t)=1, \varphi(t)=0$, there exists a function $H_{2}(t, s) \in X_{\gamma}$ such that, for all sufficiently large $t_{1} \geq t_{0}$ and $t_{2} \geq t_{1} \geq t_{0}$,

$$
\lim _{t \rightarrow \infty} \sup \frac{1}{H_{2}\left(t, t_{2}\right)_{t_{2}}}\left[\left(1-{ }_{j=1}^{n} p_{0 j}\right)^{\gamma}\left(s-t_{1}\right)^{\gamma} H_{2}(t, s)_{i=1}^{m} q_{i}(s)-\frac{\left(h_{2}(t, s)\right)^{\gamma+1} a(s)}{(\gamma+1)^{\gamma+1}}\right] d s>0
$$

Then every solution $x$ of eqn. (E) is either oscillatory or satisfies $\lim _{t \rightarrow \infty} x(t)=0$.

Proof: Assume that $x(t)$ is a positive solution of eqn. (E). Based on the condition eqn. (1.3), there exist the three possible cases (I), (II) and (III) for $t \geq t_{1}, t_{1}$ is large enough. Assume that case (I) and case (II) hold, respectively. We can obtain the conclusion of Theorem 9 by applying the proof of Theorem 8 . Now assume that case (III) holds. Consider again the function $\phi(t)$ defined by eqn. 3.16. Then we can easily deduce eqn. 3.18. Since $z^{\prime}(t)>0$ and $\sigma_{i}(t) \geq t, i=1,2, . ., m$, we obtain

$$
\phi^{\prime}(t) \leq-\left(1--_{j=1}^{n} p_{0 j}\right)_{i=1}^{\gamma m} q_{i}(t)\left(\frac{z(t)}{z^{\prime}(t)}\right)^{\gamma}-\frac{\gamma \phi^{\frac{\gamma+1}{\gamma}}(t)}{a^{\frac{1}{\gamma}}(t)} .
$$

In view of (III), we see that

$$
\left(\frac{z(t)}{z^{\prime}(t)}\right)^{\gamma} \geq\left(t-t_{1}\right)^{\gamma}
$$

This with eqn. (3.33) leads to

$$
\phi^{\prime}(t) \leq-\left(1-{ }_{j=1}^{n} p_{0 j}\right)_{i=1}^{\gamma m} q_{i}(t)\left(t-t_{1}\right)^{\gamma}-\frac{\gamma \phi^{\frac{\gamma+1}{\gamma}}(t)}{a^{\frac{1}{\gamma}}(t)} .
$$

Going through as in the proof of Theorem 7, we can easily deduce that

$$
\frac{1}{H_{2}\left(t, t_{2}\right)_{t_{2}}}{ }^{t}\left[\left(1-{ }_{j=1}^{n} p_{0 j}\right)^{\gamma}\left(s-t_{1}\right)^{\gamma} H_{2}(t, s)_{i=1}^{m} q_{i}(s)-\frac{\left(h_{2}(t, s)\right)^{\gamma+1} a(s)}{(\gamma+1)^{\gamma+1}}\right] d s \leq \phi\left(t_{2}\right),
$$

which contradicts eqn. 3.32. This completes the proof.

\section{Example 1}

Consider the differential equation

$$
\left(\left(\left(x(t)+\frac{1}{e^{\frac{1}{5}}} x\left(t-\frac{1}{5}\right)+\frac{1}{5 e^{\frac{1}{2}}} x\left(t-\frac{1}{2}\right)\right)^{\prime \prime}\right)^{3}\right)^{\prime}+\frac{3993}{125} x^{3}(t)=0, t \geq 1 .
$$

Choosing $\rho(t)=\frac{1}{t^{3}}, \varphi(t)=\frac{1}{8 t^{3}}$ and $H(t, s)=(t-s)^{2}$. It is clear that all assumptions of Theorem 6 are satisfied. Hence, every solution $x$ of eqn. (3.35) is either oscillatory or satisfies $\lim _{t \rightarrow \infty} x(t)=0$. As a matter of fact, one such solution is $x(t)=e^{-t}$.

\section{Example 2}

Consider the differential equation

$$
\left(t^{3}\left(x(t)+\frac{1}{16} x\left(\frac{t}{2}\right)+\frac{1}{108} x\left(\frac{t}{3}\right)\right)^{\prime \prime}\right)^{\prime}+42 x(t)=0, t \geq 1 .
$$

By choosing $\quad \rho(t)=\frac{1}{t}, \quad \varphi(t)=\frac{1}{2 t}, \quad H(t, s)=\left(\begin{array}{ll}t & s\end{array}\right)$ $H_{1}(t, s)=\frac{(t-s)^{2}}{s^{2}}$ and $t_{2}=t_{1} \stackrel{t}{=} t_{0}=1$. It is clear that all assumptions of Theorem 7 are satisfied. Hence, every solution $x$ of eqn. 3.36 is either oscillatory or satisfies $\lim _{t \rightarrow \infty} x(t)=0$, when $c>\frac{108}{2807}$. In fact such solution of eqn. 3.36 is $x(t)=\frac{1}{t^{3}}$.

\section{Example 3}

Consider the differential equation

$$
\left(t\left(x(t)+p_{1} x\left(\frac{t}{2}\right)\right)^{\prime \prime}\right)^{\prime}+\frac{\lambda}{t^{2}} x(t)=0, \lambda>0, t \geq 1 .
$$

By choosing $\rho(t)=1, \varphi(t)=0$ and $H(t, s)=(t-s)^{2}$. It is clear that all assumptions of Theorem 6 and Theorem 8 are satisfied. Hence, every solution $x$ of eqn. 3.37 is either oscillatory or satisfies $\lim _{t \rightarrow \infty} x(t)=0$, provided that $\lambda>0$. We note that the authors [10] proved that eqn. 3.37 is oscillatory if $\lambda>1 /\left(4 k\left(1-p_{1}\right)\right)$ for some $k \in(1 / 4,1)$ and so our result improves those [10].

\section{References}

1. Baculková B, Džurina J (2010) Oscillation of third-order neutral differential equations. Mathematical Computational Modelling 52: 215-226.

2. Baculková B, Džurina J (2010) Oscillation of third-order functional differentia equations. Electronic Journal of Qualitative Theory of Differential Equations 43 $1-10$.

3. Baculková B, Džurina J (2011) Oscillation of third-order nonlinear differential equations. Applied Mathematics Letters 24: 466-470

4. El-Sheikh MMA, Sallam RA, Mohamady N (2013) On the oscillation of thirdorder neutral delay differential equations. Applied Mathematics \& Information Sciences Letters 1: 77-80.

5. Grace SR, Agarwal RP, Pavani R, Thandapani E (2008) On the oscillation of certain third order nonlinear functional differential equations. Applied Mathematics and Computation 202: 102-112.

6. Hardy GH, Littlewood JE, Polya G (1988) Inequalities. (2nd edn.), Cambridge University.

7. Jiang J (2003) Oscillation of second order nonlinear neutral differential equations. Applied Mathematics and Computation 135: 531-540.

8. Jiang Y, Li T (2014) Asymptotic behavior of a third-order nonlinear neutral delay differential equation. Journal of Inequalities and Applications, p: 512.

9. Li T, Rogovchenko YV, Tang S (2014) Oscillation of second-order nonlinear differential equations with damping. Math Slovaca 64: 1227-1236.

10. Li T, Zhang C, Xing G (2012) Oscillation of third-order neutral delay differential equations. Abstract and Applied Analysis, pp: 1-11.

11. Michelson D (1986) Steady solutions of the Kuramoto-Sivashinsky equation. Physica D 19: 89-111.

12. Philos CG (1989) Oscillation theorems for linear differential equations of second order. Archiv der Mathematik 53: 482-492.

13. Zhong J, Ouyang Z, Zon S (2011)Oscillation criteria for a class of third-order nonlinear neutral differential equations. Journal of Applied Analysis 17: 155163. 